\title{
Einsatz des Erbium:YAG-Lasers in der korrektiven Dermatologie
}

\author{
Use of the Erbium: YAG Laser in Corrective Dermatology
}

\section{Zusammenfassung}

Die Laserablation hat zur umschriebenen oder auch flächenhaften Entfernung oberer Hautschichten im Spektrum operativ-dermatologischer Therapiemöglichkeiten eine weite Verbreitung erfahren. Insbesondere die Erbium:YAG-Lasertechnologie bietet heute die Möglichkeit einer hochpräzisen und kontrollierten Hautabtragung. Sie ist bei zahlreichen Indikationen einfach durchführbar, wenig belastend und gut steuerbar. In vielen Fällen hat die Laserablation eine mechanische Dermabrasion oder andere abtragende Techniken ersetzt. Eine schonende Gewebeabtragung ist im Rahmen ästhetisch-korrektiver Eingriffe, aber auch in atrophischer Altershaut und in kritischen Hautregionen von besonderem Nutzen, um das Risiko von Wundheilungsverzögerungen oder unerwünschter Nebenwirkungen zu minimieren.

\section{Abstract}

Laser resurfacing techniques are widely used among the spectrum of dermatosurgical procedures in order to remove circumscribed skin lesion as well as larger areas. In particular, Erbium:YAG-laser-technology enables us to perform a highly precise skin ablative work. In a variety of indications superficial skin ablation is safe, not associated with major discomfort, and easy to control. Therefore, this technique has replaced mechanical dermabrasion and other modalities of tissue removal in many indications. Careful laser ablation is of special worth in aesthetic skin surgery, also when treating disorders appearing in atrophic skin of elderly patients or in more delicate areas in order to prevent prolonged healing along with unwarranted side effects.

\section{Einleitung}

Neben den verschiedenen Lasersystemen zur Beseitigung ästhetisch störender Gefäß- oder Pigmentläsionen hat das Interesse an Geräten zur Hautabtragung in den vergangenen Jahren eine besondere Bedeutung gewonnen, lassen sich doch hiermit sowohl punktuell umschriebene wie auch flächenhaft befallene Gewebebezirke in unterschiedlicher Tiefe beseitigen. Im Vergleich zu konventionellen Techniken der umschriebenen oder großflächigen Gewebeentfernung (Dermashaving, Ringkürette, Stanzenexzision, Dermabrasion) ist ein sehr präzises und gewebeschonendes Vorgehen erzielbar.
Hierbei haben gepulste Lasertechnologien mit maximaler Gewebeabsorption zu einer Vermeidung unerwünschter thermischer Begleitschäden geführt. Mit der Einführung des gepulsten Erbium:Yttrium-Aluminium-Granat (Er:YAG)-Lasers ist dies in besonderem Maße gelungen, da dessen infrarote Strahlung bei $2940 \mathrm{~nm}$ Wellenlänge hauptsächlich von Gewebewasser und Proteinen absorbiert wird. Der Einzelpuls eines Er:YAG-Lasers entfernt bei einer Energiedichte von $5 \mathrm{~J} / \mathrm{cm}^{2}$ eine etwa $20-25 \mu \mathrm{m}$ dicke Gewebeschicht und hinterlässt eine schmale Nekrosezone von lediglich 5-15 $\mu \mathrm{m}$ [1 - 3]. Im Gegensatz hierzu verursachen die bei einer Wellenlänge von $10600 \mathrm{~nm}$ weniger gut im Gewebewasser absorbierten $\mathrm{CO}_{2}$-Laserstrahlen selbst bei gepulster Applikation thermische Begleitschädigungen. Überdies kumulie- 
ren bei $\mathrm{CO}_{2}$-Laserabtragung die Koagulationszonen mit zunehmenden Pulsfolgen bis über $100-150 \mu \mathrm{m}$, was beim Er:YAG-Laser nicht der Fall ist. Die nach forcierter Anwendung des gepulsten $\mathrm{CO}_{2}$-Lasers beschriebenen Langzeit-Komplikationen, wie persistierende Erytheme oder anhaltende Depigmentierungen, sind daher eher vermeidbar. Allerdings stellen sich analog einer Dermabrasion gleichartige Risiken in Abhängigkeit von der $\mathrm{Ab}$ tragungstiefe ein. So lässt sich der Er:YAG-Laser zwar zu einer sehr schonenden Abtragung der Haut einsetzen, kann aber in Abhängigkeit der Bestrahlungsparameter und Pulszahlen ebenso zu tiefen Defekten (flächenhaft analog einer tiefen Dermabrasion oder umschrieben analog einer Hautstanzung) mit entsprechenden Risiken der Narbenbildung führen.

Verschiedene Modifikationen (Verlängerung der Pulsdauer, subablative Pulsserien) des Er:YAG-Lasers ermöglichen zudem bei entsprechenden Indikationen erforderlichenfalls auch die Erzeugung rein thermischer Effekte [4,5]. Neuere Systeme sind daher vielseitig einsetzbar, indem sie wahlweise sowohl ein rein ablatives als auch ein koagulatives Vorgehen ermöglichen. Dies gestattet punktuelle Ablationen mit tieferreichenden kraterförmigen Defekten (z.B. Entfernung von Syringomen), rasch durchführbare Laserabrasionen größerer Hautbezirke (Laser-Peeling, Laser-Resurfacing) und erforderlichenfalls auch die zusätzliche oder reine Koagulation (z.B. Rhinophym-Op, Blepharoplastik, „Subsurfacing“).

\section{Indikationen in der korrektiv-ästhetischen Dermatologie}

Sowohl umschriebene als auch ausgedehntere Veränderungen, wie sie zum Beispiel bei einer aktinischen Schädigung der Haut vorkommen, eignen sich für eine rasche und schrittweise Laserablation. Auch punktförmige Läsionen, die bis in die tiefere Dermis hineinreichen, können mit Hilfe eines fokussierten Strahls gezielt kraterförmig abladiert werden, so dass die entstehende Narbe möglichst klein oder gänzlich unauffällig wird [6].

Ungeachtet der Indikation ist die Ablation durch den Er:YAG-Laser auch an anatomisch kritischen Stellen vorteilhaft. Vor allem die Haut der Augenlider, zervikal, im Dekolletébereich und an den Handrücken sollte im Rahmen eines Resurfacings nur einer schonenden Ablation unterzogen werden. Tiefere Schädigungen sind hier unbedingt zu vermeiden, da in diesen Bereichen gerade die atrophische Haut älterer Patienten dünner ist und im Vergleich zum Gesicht hier auch die reduzierte Anzahl an Adnexstrukturen eine Reepithelisierung erschwert.

\section{Entfernung aktinischer Spätfolgen}

Die Laserablation verschiedener Folgeerscheinungen einer chronisch UV-Licht-geschädigten Haut mit Hilfe des Er:YAG-Lasers hat im Vergleich zum Chemical Peeling oder zur Dermabrasion, aber auch zur thermischen Abtragung mit gepulsten $\mathrm{CO}_{2}$-Lasern aufgrund der besseren Steuerbarkeit mit eher abschätzbaren Risiken der umschriebenen und in der Tiefenausdehnung kalkulierbaren Wundflächen einen zunehmenden Stellenwert erfahren. Bevorzugt wird der Er:YAG-Laser zur Behandlung oberflächlicher kleiner Faltenbildungen im Rahmen der kutanen Elastose, von Zysten und Komedonen bei M. Favre-Racouchot oder zur Entfernung solarer Lentigines eingesetzt [7]. Die Hauptvorteile des gepulsten Er:YAG-Lasers liegen bei diesen Indikationen in der Möglichkeit einer sehr superfiziellen Gewebeentfernung mit höchstmöglicher Präzision in der ablativen Technik. Diese kontrollierte Abtragung zusammen mit der Vermeidung thermischer Begleitschäden ermöglicht nicht nur die Behandlung von Hautarealen oder Hauttypen mit höherem Komplikationsrisiko, sondern trägt auch zur möglichst raschen Wundheilung im Rahmen korrektiv-ästhetischer Anwendungen bei.

Bei Morbus Favre-Racouchot galt früher die Dermabrasion als Therapieoption. Wie bei anderen Indikationen hat sich in unseren Händen hier der Er:YAG-Laser als überlegene Alternative durchgesetzt, da in den befallenen periorbitalen und temporalen Hautbezirken eine bessere Kontrolle und erforderlichenfalls auch ein punktuell tieferes Arbeiten möglich ist.

Zur Enfernung solarer Lentigines respektiver seniler Lentigines, die feingeweblich sehr oberflächlichen seborrhoischen Keratosen entsprechen, bietet die umschriebene superfizielle Ablation mit dem Er:YAG-Laser durchaus eine Alternative zu gütegeschalteten Lasersystemen, wie sie zur selektiven Pigmentphotothermolyse eingesetzt werden (Nd:YAG-Laser, Rubinlaser, Alexandritlaser). Voraussetzung ist allerdings ein sehr oberflächliches Abtragen, bei dem mit wenigen Pulsen möglichst nur das Epithel entfernt wird, um gerade in der atrophischen Altershaut (insbesondere am Handrücken) Narben oder Spätdepigmentierungen zu verhindern.

Zur Behandlung feiner Faltenbildungen in elastotisch veränderter Haut sind gegenwärtig gepulste $\mathrm{CO}_{2}$-Laser, Er:YAG-Laser im ablativen Modus, Er:YAG-Laser mit zusätzlichem thermischen Modus und auch Kombinationsgeräte aus beiden Lasertypen im Einsatz [8 - 11]. Im Rahmen der $\mathrm{CO}_{2}$-Laseranwendung oder auch bei thermisch wirkenden Kombinationen des Er:YAG-Lasers wird postuliert, dass thermische Begleitwirkungen, die eine während der Behandlung bereits erkennbare Straffung der Hautoberfläche („shrinkage“) mit anhaltender Glättung während der verlängerten Ödemphase und letztlich im Rahmen der später verstärkt einsetzenden dermalen Fibrose bewirken, für den Therapieerfolg vorteilhaft sind. Insgesamt scheint es jedoch, vorausgesetzt man vergleicht eine Ablation des Er:YAG-Lasers mit einer $\mathrm{CO}_{2}$-Vaporisation gleicher Gesamttiefe, dass sich unter diesen Bedingungen die Ergebnisse insbesondere bei feinen oberflächlichen Fältchen mehr oder weniger ähneln und damit eher die Gesamttiefe des Hautschadens (Abtragung plus thermische Nekrosezone) und nicht die Modalitäten an sich die Wirksamkeit und Risiken des abtragenden Verfahrens bestimmen [12-14]. So bleibt umstritten, ob der ausgeprägtere Hitzeschaden durch gepulste $\mathrm{CO}_{2}$-Laser dessen Einsatz zumal in kritischen Hautregionen oder bei weniger ausgeprägter Elastose gerechtfertigt erscheinen lassen.

Abgesehen von der kontroversen Diskussion um die tatsächliche Rolle der jeweiligen Resurfacing-Techniken bei lichtgealterter Haut steht außer Frage, dass der Er:YAG-Laser bei der Behandlung anatomisch kritischer Lokalisationen und bei Patienten mit hinsichtlich Pigmentverschiebungen gefährdetem Hauttyp Vorzüge aufweist [15 - 17]. Daher findet er bevorzugt in der Perioralund der Periorbitalregion auch in Kombination mit der Blepharoplastik Anwendung. Die hohe Präzision der Ablation des Er:YAG- 
Lasers zusammen mit der gewebeschonenden Anwendung kann in diesen Fällen weder durch eine Dermabrasion noch durch eine $\mathrm{CO}_{2}$-Vaporisation erreicht werden. Eine Alternative ist der Einsatz des Er:YAG-Lasers im subablativen Modus im Sinne des sog. „Subsurfacing“. Hierbei wird postuliert, durch eine rein thermische Wirkung im oberen Corium eine Fibroblastenstimulation mit Induktion der Kollagensynthese und einer feinen Fibrose das Oberflächenrelief der aktinisch geschädigten Haut glatter erscheinen zu lassen. Wie bei allen hierzu eingesetzten Systemen sind aber gegenwärtig etwaige Erfolge klinisch schwierig zu objektivieren.

\section{Laserablation epithelialer Neu- und Fehlbildungen}

Unter den umschriebeneren Läsionen können insbesondere flache seborrhoische Keratosen (inklusive der oben beschriebenen Lentigo senilis als oberflächliche Variante), vor allem auch an empfindlicheren Lokalisationen, einfach und präzise abgetragen werden.

Mitunter lediglich ästhetisch störende kleinere und papulös aggregierte epidermale Nävi in Problemlokalisationen oder optisch exponierten Arealen (z. B. Lidbereich, Stirn) sind ebenfalls gut für eine Laserablation geeignet. Im Gegensatz zur flächenhaften mechanischen Dermabrasion, kann bei dieser Indikation gerade im Bereich der kleinen Papeln punktuell tiefer abladiert werden, um Rezidiven vorzubeugen [18].

\section{Laserablation von Veränderungen der Adnexe und der Dermis}

Dermale und adnexielle Veränderungen, wie Syringome, Talgdrüsenhyperplasien oder Xanthelasmen, eignen sich ebenfalls gut für eine schrittweise und schonende Ablation, doch auch andere störende Läsionen (z.B. kleinere Fibrome) lassen sich gezielt abtragen [19].

Die Entfernung von Syringomen mittels vaporisierender Lasersysteme wurde bereits verschiedentlich propagiert. Aufgrund der Vermeidung von thermischen Schädigungen im feinen Lidhautbereich ist aber eine präzise Laserablation vorteilhaft. Hierbei können mit dem Er:YAG-Laser punktuelle Ablationskanäle analog feinster Bohrlöcher bis in das Corium erzielt werden, ohne dass bei tieferer Ablation die Hitzeschädigung am Wundgrund zunimmt, wie dies bei analoger Vorgehensweise mit vaporisierenden Systemen der Fall wäre [20]. Die Sekundärheilung im Unterlidbereich liefert in der Regel bei einer Vorgehensweise in mehreren Sitzungen sehr gute Ergebnisse mit allenfalls passager sichtbaren punktuellen Rötungen und später nahezu unsichtbaren Närbchen. Ein flächenhaftes Abtragen im Sinne des Resurfacing“ der Lidhaut ist bei dieser Indikation nicht indiziert, da bei entsprechender Abtragungstiefe bis zum Erreichen der korial lokalisierten Syringome mit entsprechenden Narbenbildungen zu rechnen ist.

Auch die Entfernung von Xanthelasmen wird vornehmlich aus kosmetischen Gründen gewünscht. Eher spindelig oder streifenförmig ausgerichtete Läsionen können in der Regel problemlos exzidiert oder mittels tangentialer Scherenabtragung entfernt werden, wobei in solchen Fällen bei Befall der Oberlidhaut die Exzision ggf. auch mit einer Blepharoplastik kombiniert werden kann. Bei Vorliegen breiterer Plaques oder multipel aggregierter
Varianten bevorzugen wir allerdings die schrittweise Laserablation mit einer sekundären Wundheilung der entstandenen Defekte. In der Regel liefert diese Vorgehensweise gute Ergebnisse.

Ebenso stellt die Laserablation für die Entfernung der oft störenden älteren dermalen Nävuszellnävi der Gesichtshaut („weiche Nävi“) eine Alternative zum sog. Dermashaving oder der elektrokaustischen Abtragung dar. Im Gegensatz zum tangentialen Skalpellschnitt oder dem Abtragen mit einer Ringkurette, kann hier wesentlich präziser und umschriebener, punktuell auch tiefer reichend, abgetragen werden [6]. Anders als bei elektrokaustischer Abtragung oder Laservaporisation lassen sich thermische Begleiteffekte vermeiden.

Weiterhin ist die Ablation des Gewebes bei einzelnen oder multiplen Talgdrüsenhyperplasien ebenso wie beim Rhinophym möglich. Talgdrüsenhyperplasien können sehr einfach schrittweise abladiert werden. Um das Risiko atrophischer Narbenbildungen zu minimieren, sollten hierbei tiefere kraterförmige Läsionen vermieden werden. Zur Rhinophymentfernung kann der Erbium-Laser bei weniger gefäßreichen Knotenbildungen alternativ zur elektrischen Schlingenabtragung oder der $\mathrm{CO}_{2}$-LaserVaporisation eingesetzt werden. Hier sind besonders die mit einer Hämostasefunktion ausgestatteten Systeme vorteilhaft.

\section{Laserablation von Narben}

Der Einsatz des Er:YAG-Lasers kann bei Aknenarben, zur Korrektur von hypertrophen Narben, aber auch zur Angleichung von Stufenbildungen oder Unregelmäßigkeiten im Verlauf von Operationsnarben hilfreich sein [21].

Im Falle der Behandlung von Aknenarben der Gesichtshaut setzen wir die Er:YAG-Laserablation seit vielen Jahren routinemäßig als Alternative zur Dermabrasion ein. Hierbei werden jeweils symmetrisch gesamte ästhetische Einheiten, bevorzugt die Wangenregionen, abgetragen. Gegenüber der Dermabrasion hat die Methode den Vorteil, dass ein wesentlich homogeneres Abtragen mit gleichförmiger Tiefe in Abhängigkeit der gewählten Bestrahlungsparameter und Pulsserien gewährleistet bleibt und dies unabhängig von der Lokalisation problemlos möglich ist. Insbesondere in den bei Dermabrasionen kritischen Regionen der perioralen und periorbitalen Haut ebenso wie im Bereich der Nase können so Risiken der mechanischen Abtragung und auch ein akzidentelles zu tiefes Dermabradieren vermieden werden. Ebenso lassen sich umschrieben an einzelnen Narben zusätzliche Ablationen bewerkstelligen, um beispielsweise scharfkantig imponierende Ränder einzuebnen. Auch ist in den Randbereichen ein stufenloser Übergang durch Verwendung von Einzelpulsserien am Übergang zur unbehandelten Haut zur Vermeidung einer sichtbaren Demarkationslinie besser als mit der Dermabrasion möglich. Erfolge der Er:YAG-Laserablation in der Behandlung von Aknenarben wurden von verschiedenen Untersuchern und bei unterschiedlichen Hauttypen bestätigt, wobei im Gegensatz zum $\mathrm{CO}_{2}$-Laserresurfacing oder auch einer kombinierten Anwendung beider Applikationen die postoperativen Erytheme und Wundheilungsphasen kürzer waren [22]. Insbesondere gilt dies im Falle eines oberflächlichen „Laserpeelings“, wobei nur das Epithel mit 2 bis 4 Pulsserien (gesamt ca. $10-20$ Joule $/ \mathrm{cm}^{2}$ ) abgetragen wird. Dieses Prozedere lässt sich wiederholen, wobei wir die Patienten in jährlichen Abständen in den Wintermonaten 
Tab. 1 Indikationen des Er:YAG-Lasers in der korrektiv-ästhetischen Dermatologie

\section{Aktinische Spätschäden}

oberflächliche Fältchen im Rahmen der Elastosis cutis

M. Favre-Racouchot

2. Epidermale Neu- und Fehlbildungen

epidermale Nävi

senile Lentigines

seborrhoische Warzen

3. Melanozytäre Neu- und Fehlbildungen

kongenitale Riesennävi

dermale Nävi („weiche Nävi“)

\section{Neubildungen im Bereich der Adnexe und Dermis}

Syringome

Xanthelasmen

Fibrome

Talgdrüsenhyperplasien

Rhinophym

\section{Narben}

Aknenarben

postoperative Narben

\section{Sonstige}

laser-assistierte Haartransplantationen bei androgenetischer Alopezie

Osteoma cutis

multikolorierte Tätowierungen

therapieren. Wie auch bei der Dermabrasio werden die bei Aknenarben erzielten Erfolge subjektiv vom Patienten oft besser eingeschätzt als dies objektiv nachvollziehbar erscheint. In der Regel ist das Prozedere in Abhängigkeit des Narbenbildes mit weiteren Techniken (z.B. Stanzenelevationen, Exzisionen) zu kombinieren.

Bei hypertrophen Narben und insbesondere bei Keloiden ist die Abtragung analog der Vaporisation mit dem $\mathrm{CO}_{2}$-Laser oder einem Shaving allenfalls in Kombination mit anderen Verfahren Erfolg versprechend. Hingegen lässt sich bei abgestuften Narben oder leicht hypertrophen schmalen Operationsnarben in geeigneten Fällen eine gewisse Glättung nach Laserablation erzielen, so dass hier ein Therapieversuch gerechtfertigt erscheint.

\section{Sonstige korrektiv-ästhetische Indikationen}

Unter den zahlreichen übrigen Indikationen, die für eine flächenhafte oder umschriebene Laserablation in Betracht kommen, sind unter ästhetisch-korrektiven Aspekten noch einige seltenere Indikationen zu erwähnen.

Hierzu zählt der Einsatz des Er:YAG-Lasers im Rahmen der laserassistierten Haartransplantation. Hier verwenden wir ihn als geeignetes Instrument zur Erzielung schräg eingebrachter Empfängerlöcher bei narbigen Formen der Alopezie, wo es auch bei tiefen Kraterläsionen kaum zu Blutungen kommt, andererseits aber die bei Verwendung eines $\mathrm{CO}_{2}$-Lasers entstehende Nekrosezone um den Empfängerkanal-Behinderung der Transplantateinheilung vermieden wird [23].

Eine weitere Indikation stellen farbige Tätowierungen dar, deren Pigmentkomponenten einer selektiven Pigmentphotothermolyse (z.B. gütesschaltete Rubin-, Alexandrit- oder Nd:YAG-Laser) nicht oder nur teilweise zugänglich und die aufgrund ihrer Ausdehnung oder Konfiguration für eine alternativ in Erwägung zu ziehende Exzision ungeeignet sind. Im Gegensatz zu der in solchen Fällen ebenfalls in Betracht kommenden Dermabrasion hat der Er:YAG-Laser den Vorteil, dass nach anfänglicher flächenhafter Freilegung und oberflächlicher Abtragung des pigmenthaltigen Coriums nur noch an umschriebenen Stellen in die Tiefe weiter abgetragen werden muss, wo sich Pigmentpartikel zeigen. Eine derartig punktuelle und präzise Gewebeabtragung ist mit der Dermabrasion nicht möglich.

Unter den selteneren Indikationen sei das ebenfalls in erster Linie kosmetisch störende Krankheitsbild der miliaren Osteomata cutis genannt, wo ebenfalls eine laserassistierte Entfernung mit dem Er:YAG-Laser möglich ist. Auch hier gestattet die punktuelle Ablation im Gegensatz zu anderen hautabtragenden Techniken ein präzises Freilegen und z.T. der kleinen korial gelegenen Neubildungen, die dann mittels feiner Pinzetten oder auch durch tiefere Ablation entfernt werden können [24] (Tab.1).

\section{Literatur}

${ }^{1}$ Hohenleutner U, Hohenleutner S, Baumler W, Landthaler M. Fast and effective skin ablation with Er:YAG laser: determination of ablation rates and thermal damage zones. Lasers Surg Med 1997; 20: $242-247$

${ }^{2}$ Kaufmann R, Hibst R. Pulsed Erbium:YAG and 308 nm UV-Excimer laser: an in vitro and vivo study of skin-ablative effects. Lasers Surg Med 1989; 9: $132-140$

${ }^{3}$ Kaufmann R, Hartmann A, Hibst R. Cutting and skin-ablative properties of pulsed mid-infrared laser surgery. J Dermatol Surg Oncol 1994; 20: $112-118$

${ }^{4}$ Hibst R, Stock K, Kaufmann R. Ablation and controlled heating of skin with the Er:YAG Laser. Lasers Surg Med 1997; 9: 40

${ }^{5}$ Weinstein C. Modulated dual mode erbium $/ \mathrm{CO}_{2}$ lasers for the treatment of acne scars. J Cutaneous Laser Therapy 1999; 1: 203-208

${ }^{6}$ Kaufmann R. Comparison of different procedures for the treatment of benign and malign skin tumors. Min Invas Ther \& Allied Technol 1998; 7: $511-517$

${ }^{7}$ Kaufmann R. Role of Erbium:YAG Laser in the treatment of the aged skin. Clin Exp Dermatol 2001; 26: 631 -636

${ }^{8}$ Fitzpatrick RE, Goldman MP, Satur NM, Tope W. Pulsed carbon dioxide laser resurfacing of photoaged facial skin. Arch Dermatol 1996; 132: $395-402$

${ }^{9}$ Teikemeier G, Goldberg DJ. Skin resurfacing with the erbium:YAG laser. Dermatol Surg 1997; 23: 685-687

${ }^{10}$ Perez MI, Bank DE, Silvers D. Skin resurfacing of the face with the Erbium:YAG laser. Dermatol Surg 1998; 24: 653-659

${ }^{11}$ Goldman MP, Marchell N, Fitzpatrick RE. Laser skin resurfacing of the face with a combined $\mathrm{CO}_{2}$ /Er:YAG Laser. Dermatol Surg 2000; 26 : $102-104$

12 Adrian RM. Pulsed carbon dioxide and erbium-YAG laser resurfacing: a comparative clinical study. J Cutan Laser Ther 1999; 1: 29-35

${ }^{13}$ Fleming D. Controversies in skin resurfacing: the role of the erbium. J Cutan Laser Ther 1999; 1: 15-21

${ }^{14}$ Kaufmann R, Fleming D. Erbium:YAG laser rejuvenation. In: Lowe NJ (Ed). Textbook of facial rejuvenation. London: M. Dunitz, 2002: $101-122$

15 Jimenez G, Spencer JM. Erbium:YAG laser resurfacing of the hands, arms, and neck. Dermatol Surg 1999; 25: 831 -834

${ }^{16}$ Weiss RA, Harrington AC, Pfau RC, Weiss MA, Marwaha S. Periorbital skin resurfacing using high energy erbium:YAG laser: results in 50 patients. Laser Surg Med 1999; 24: 81-86

${ }^{17}$ Polnikorn N, Goldberg DJ, Suwanchinda A, Ng SW. Erbium:YAG Laser Resurfacing in Asians. Dermatol Surg 1998; 24: $1303-1307$

${ }^{18}$ Kaufmann R, Hibst R. Pulsed $2.94 \mu \mathrm{m}$ Erbium-YAG laser skin ablation: experimental results and first clinical application. Clin Exp Dermatol 1990; 15: 389-393

${ }^{19}$ Kaufmann R, Hibst R. Clinical evaluation of Er:YAG Lasers in cutaneous surgery. Lasers Surg Med 1996; 19: 324-330

${ }^{20}$ Kaufmann R, Beier C. Erbium:YAG laser therapy of skin lesions. Medical Laser application 2001; 16: 252-263 
${ }^{21}$ Kaufmann R, Beier C. Narben-Korrekturmöglichkeiten mit dem Laser. In: Mang WL, Kokoschka EM (Hrsg). Ästhetische Chirurgie, Band II. Reinbek: Einhorn Presse Verlag, 1998: 20-25

${ }^{22}$ Kye YC. Resurfacing of pitted facial scars with the Er:YAG laser. Dermatol Surg 1997; 23: 880-883
${ }^{23}$ Podda M, Spieth K, Kaufmann R. Er:YAG laser-assisted hair transplantation in cicatricial alopecia. Dermatol Surg 2000; 26: 1010 - 1014

24 Ochsendorf FR, Kaufmann R. Erbium:YAG laser assisted treatment of miliary osteoma cutis. Br J Dermatol 1998; 138: 371 - 372

\section{Buchbesprechung}

\section{Photodermatosen und Lichtreaktionen der Haut} Hölzle E.

376 S., 89 Abb. Wissenschaftliche Verlagsgesellschaft, Stuttgart 2003, Geb. 118.- . ISBN 3-8047-1890-6

Um es vorwegzunehmen - endlich ist die längst fällige, in Jahrzehnten erfolgreicher Forschung und ärztlicher Erfahrung sorgfältig recherchierte Monographie zum Thema Wirkungen optischer Strahlung auf die Haut des Menschen und den Rest des Organismus vorgelegt worden. Der Verfasser ist ein Kenner der Materie, dem das Kunststück gelang, in allgemein verständlicher Fachsprache einen Bogen von den unerläßlichen physikalischen und chemischen Fakten über die erforderlichen technischen, physiologischen, biologischen, morphologischen und immunologischen Aspekte bis hin zur besonnten Haut zu schlagen. Die hier interessierenden Facetten dieser sehr unterschiedlichen Fachgebiete werden souverän und didaktisch geschickt auf nicht weniger als 376 Seiten präsentiert.

Innerhalb der Dermatologie hat sich seit einigen Jahren die Subspezialität „Photodermatologie“ etabliert. So ist es nur folgerichtig, wenn Hölzle die in diese Subspezialität fallenden Dermatosen als „Photodermatosen“ bezeichnet. Daneben ist aber auch von Lichtdermatosen und Lichtreaktionen die Rede. Das dahinter stehende nomenklatorische Dilemma ergab sich, weil Begriffe wie „Licht“ und „Photo“, aber auch „optisch“ zumindest etymologisch an das Sehen gebunden sind und deshalb streng genommen nicht auf die unsichtbare ultraviolette bzw. infrarote Strahlung angewendet werden dürften. Streng genommen sollte der Begriff „optische Strahlung“ verwendet werden. Darunter fallen alle elektromagnetischen Strahlen, die mit optischen Mitteln - beispielsweise Spiegel oder Linsen - manipuliert werden können, d. h. Licht, Ultraviolett und Infrarot, nicht aber Röntgenstrahlen. Doch auch dieser wohl definierte Begriff hat seine Grenzen. Beispielsweise wäre das Umbenennen von Photodermatologie in Optodermatologie oder von Photodermatosen in Optodermatosen nicht zu empfehlen. Und so sollten wir nach wie vor den fachsprachlichen Gebrauch der Begriffe „Licht“ und „Photo“ im weiteren Sinne dieser Worte tolerieren.
Die vorliegende Monographie richtet sich nicht nur an Photodermatologen, sondern ist von großem Wert für alle, die mit Fragen über Licht und Gesundheit konfrontiert werden. Angesichts sehr unterschiedlicher Interessenlagen und der anhaltenden Vorliebe der Medien für die dunkle Seite der Sonne müßten das sehr viele Personen sein. Und sehr viele sollten die ausgewogenen, gut aufbereiteten und alles Wesentliche referierenden Kapitel lesen: Physikalische Grundlagen (17 Seiten) - Biologische Wirkungen optischer Strahlung (33 Seiten) - Akute Lichtschäden (18 Seiten) - Chronische Lichtschäden (27 Seiten) - Idiopathische Lichtdermatosen (91 Seiten) - Chemische Photosensibilisierung (32 Seiten) - Porphyrien (33 Seiten) - Photobiologie des Lupus erythematodes (14 Seiten) - Lichtaggravierung anderer Dermatosen (7 Seiten) - Diagnostisches Vorgehen bei Photodermatosen (25 Seiten) - Lichtschutz (15 Seiten).

Angesichts der referierten Wissensfülle kann hier auf Details kaum eingegangen werden. Es besticht die gekonnte Mischung von Grundlagenwissen und sehr praktischen Anleitungen und Tips. Als energiereichste und bislang am besten untersuchte Komponente der optischen Strahlung steht das Ultraviolett im Vordergrund der Ausführungen, hierbei insbesondere die schädigenden Auswirkungen wie vorzeitige Hautalterung und Karzinogenese. Der Verfasser begnügt sich nicht mit der Darstellung des aktuellen Wissens, sondern bespricht in den jeweiligen Kapiteln auch probate wie spekulative Möglichkeiten der Prophylaxe und Therapie. Das reicht von der Photoreparatur durch Infrarotstrahlen bis zum systemischen Lichtschutz.

Besonders lesenswert sind die Ausführungen über polymorphe Lichtdermatose. Hier drückt sich nicht nur die wohl einmalige Expertise des Autors aus, man verspürt förmlich seine Begeisterung für die Pathogenese, Diagnostik und Therapie dieser oft nur oberflächlich diagnostizierten wie therapierten Erkrankung. Zusammengefasst kann dem Verfasser und den Käufern des Buches nur gratuliert werden.

H. Meffert, Berlin 